\title{
Analysis of the Mechanical Characteristics of the Switch through the Current Waveform of Coil
}

\author{
Gao Qiang1, a, Zhang Guangming ${ }^{1}$, Liu Qi ${ }^{1}$, Shi Lin'1, Yuan Fang ${ }^{1}$, Jianshe \\ Yang $^{1}$, Jianchun $\mathrm{YaO}^{1}$, Wang Maojun ${ }^{1}$ and Zhong Dantian ${ }^{1}$ \\ 1 State Grid Liaoning Electric Power Research Institute, Liaoning, China \\ Ayao_jc@126.com
}

\section{Keywords: Mechanical characteristics, Switch, Current waveform}

\begin{abstract}
As to diagnose the circuit-breaker defect, we used to take the exit operation method, lacking of effective method for detecting the circuit-breaker operating state. Through the study of the two loop closing current, we found the corresponding relationship between two current and mechanical failure. When the switch occurred mechanical fault, the current waveform of the two circuit distorted, and there was a typical fault wave type atlas. This paper describes the development of circuit breaker detection method, and focus on some detection method of the mechanical properties of off-line H.V. circuit breakers, which here are used to capture the current waveform of opening/closing coils.
\end{abstract}

\section{Introduction}

One of the most critical devices on any electricity network is the circuit breaker which can be described as the 'silent sentinel', standing guard over the network and only being called into action when a fault occurs.

According to statistics, $60 \%-70 \%$ of the circuit-breaker defect or malfunction is caused by the circuit-breaker operating mechanism, including aging mechanism unsmooth, grease solidification, spring aging, connecting rod drive shaft fracture, control voltage instability, etc.

There are many methods of detecting the state of the circuit-breaker operating mechanism, such as infrared, ultraviolet, Partial Discharge (including UHF, ultrasound, and transient low voltage) and SF6 composition, etc. The principles of these methods are different, and the difficulty and costs of on-spot implementation are also different. But these are only for the electrical contact defects and the insulating parts (medium) defect diagnosis. There is no effective method for detecting the circuit-breaker operating state.

Current method of detecting circuit-breaker operating mechanism is to extract feature quantity and make comparison of signals, when a characterizing signal changed from normal circumstances, it might run out of order. And it requires experiences as well as theory to make analysis of the types and location of the causes of the fault.

Currently, in foreign countries, such as the United States and the Great Britain, they have extensively used circuit-breaker circuit control waveform detection technology to diagnose the status of circuit-breaker operating mechanism, guiding the circuit-breaker status maintenance strategy. In China, some GIS had installed online monitoring devices. But it lacks follow-up studies, yet the fault mechanism and diagnostic criteria are unclear, not forming a basic database. Besides, there is no calibration standard and example of application.

We know that there is very important information imbedding the varied waveform, when the coil current changes with time, in the process of circuit-breaker open and close.

The current waveform of solenoid coil contains much information. It reflects the working status of solenoid itself as well as the lock or the valve or the interlocking contacts controlled by it in the process of operation, such as the delay or tripping in the iron core movement mechanism, the changing condition of energy release mechanical load, the coil status (e.g., whether the resistance is normal), and the status of the lock and valve connecting with the iron core lever. 


\section{Process of Testing Mechanical Properties of High-voltage Circuit-breaker}

After the installment or overhaul of high-voltage circuit-breaker, we need to test its mechanical parameters according to relevant rules and requirements in order to guarantee its safe operation. The parameters include many aspects, such as opening (closing) time, simultaneity of opening (closing) time, opening range, over-travel, opening (closing) speed, average speed and maximum speed.

In early traditional testing methods, overhaul testing staffs always use electrical stop watch, synchronous lamp, light beam oscillograph, electromagnetic vibrator and drum chronograph. However, this kind of equipment is hard to be transported, and these methods have many disadvantages. They need a large area, complicated wirings and prolonged overhaul work. Moreover, reading the recorded data needs manual handling and calculation, and these methods have big measuring errors.

In late 1980s and early 1990s, the application of circuit engineering of analog digitals has developed the mechanical characteristic testing instrument of container-type circuit-breaker. This kind of instrument incorporates partial measuring functions of the instruments mentioned above, simplifying testing wiring and operations, and it is easy to carry. While it still applies traditional techniques and testing methods. For example, it uses silicon-controlled dc velometer to control circuit and consists of gate-controlled circuit, timing circuit and synchronous lamp. At the same time, it has low automation, big measuring errors and weak functions. Moreover, with the development of electricity utility, the voltage of transmission line is higher and the fractures of high-voltage circuit-breaker are increasing. Therefore, this kind of testing instrument is unable to meet the requirements in measuring the high-voltage switches of over $110 \mathrm{kV}$, because it can only simultaneously test four fractures.

With the development of micro-electronic technique and the wide application of microcomputer technology at home and abroad, testing instrument of measuring mechanical properties by microcomputer high-voltage switches has appeared in 1990s. The researches on this kind of instrument appeared in foreign countries at the beginning, and currently there are many mature products with complete functions and high anti-disturbance properties, such as the testing system of TR-3000 digital circuit-breaker developed by American DOBLE Company. In China, there firstly appeared the products using Z-80 single-board computer as microprocessor and then the products which use 8031 single chip computer as microprocessor; now, there are some productsusing 8098 or INTEL80C196 single chip computer as microprocessors. The microcomputer system using Z-80 single chip computer as microprocessor has big errors in measuring speed, low accuracy in measuring total run and over-travel of conducting rod, which is unable to reach the measuring accuracies for speed, distance and over-travel. Therefore, it is obsolescent because of MCS-51 single chip computer. The measuring instrument of high-voltage switches applying various sensors as distance sensor and 8031 single chip computer as microprocessor appearing in the mid of 1990s has many advantages contrasted with many previous instruments. For example, its time error is less than $0.1 \mathrm{~ms}$, travel error is less than $1 \mathrm{~mm}$ and speed error is less than $0.01 \mathrm{~m} / \mathrm{s}$. While its wiring and operations are also complicated. The overhaul testing staffs need to read the instructions carefully before using it, but it still led many staffs to make measuring errors or damage the instruments in transformer substation because of inappropriate wiring or usage. Therefore, it still needs to be improved. At present, there are many institutions and manufacturers who are improving this kind of products, expecting to apply them in many circuit-breakers, improving measuring accuracy, systematic stability, anti-disturbance property and perfecting their operations.

These tests of measuring high-voltage circuit-breaker mentioned above mainly are preventive tests combining with periodic overhaul during the period of equipment interchange and power outage in order to change parts and test the mechanical properties of operating mechanism. Our experience proves that frequent operations and excessive overhaul will lower the reliability of high-voltage circuit-breaker. Therefore, it is very important to diagnose or online monitor the situation of high-voltage circuit-breaker, know its operation situation and operating characteristic and changing trend in order to improve its reliability. Moreover, the power system is developing toward high-voltage and high-capacity, and power outage has greatly affected the life and production. The 
safe operation of power equipment is increasingly important. Therefore, it needs to conduct real-time or periodic online monitoring to monitor the operating condition of power equipment, so as to take preventive measures to avoid power outage. At present, some developed countries have more mature techniques in diagnosing the situation of high-voltage switches and some sections in China are also working on this area.

\section{Offline Microcomputer Instrument of Measuring Mechanical Properties of High-voltage Circuit-breaker}

We can get the starting time of circuit-breaker through testing its loop current. Through testing the switching value of fractures, we can know whether the moving and static contacts are contacting with each other or not, and the first changing time of the switching value of fractures is the opening/closing time. The period between opening/closing moment and actuation time is the opening/closing time, and the time-interval of opening/closing moment of fractures is asynchronous time. According to the changes of switching value of fractures, we also can know the oscillation situation of circuit-breaker. Testing the switching value of CPU can apply software timing interrupt mode or pulse interrupt mode. We need to expand parallel port, because we aim to test 12 fractures. Different microprocessors, different interrupt modes and different software programming will directly affect the accuracy of measuring time.

Operate within its composite tripping time (relay trip time + circuit breaker main contact opening time) to ensure correct discrimination with upstream circuit breakers and therefore minimise the number of customers disconnected during a fault operation. A slow tripping circuit breaker will not only result in unnecessary disruptions to electricity supplies but maintaining high fault currents for extended durations can stress the network and cause damage to plant and equipment. Often the problem that caused a slow trip is temporarily cleared during the first trip operation because the stickion in the mechanism is freed up. Traditional diagnostic testing procedures usually require the circuit breaker to be isolated and removed from service, therefore not only has the opportunity to detect the cause of the defect been missed but also the tests do not focus on the condition of the operating mechanism apart from overall speed of operation.

Conventional testing has normally required removing the circuit breaker from service. However, isolating the circuit breaker is time consuming as it requires a planned outage involving the issue of switching instructions, safety documents and will often involve several engineers or technicians onsite. This process resulted in vital information relating to the first trip not being captured and often the problem that caused a slow trip in a circuit breaker was temporarily cleared during this first trip When a slow trip operation occurred the focus tended to be on timing tests to determine if the problem lay within the protection relay or the circuit breaker operating mechanism. Rarely were the problems due to slow operation of the protection relay especially if they were the electronic or micro processor type. Occasionally problems occurred with the older electro-mechanical type relays due to friction in the moving parts such as the induction disc which would be revealed during secondary injection tests. Timing of the circuit breaker main contacts from initiation of a trip to main contact opening merely indicated the circuit breaker was operating within its design specification. Therefore the initial tests were inconclusive, so the focus then turned to inspecting the main operating mechanism and lubricating the numerous mechanical components. In some cases this further compounded the problem as the wrong type of lubricant was used, especially with the advent of spray penetrants which could be liberally applied. This caused an organic chemical reaction between the solvent and propellant (within the penetrant) and the soap (within the grease), resulting in varnish which caused the mechanism to 'stick'. So instead of fixing the problem that caused the slow trip, the effort in testing and maintaining the circuit breaker was largely counterproductive. 


\section{Analysis of a Trip Coil Profile}

The trip coil has an electric and magnetic circuit due to the action of a plunger moving within an energised coil. This electro-magnetic circuit has reluctance in the magnetic part as well as inductance and resistance in the electrical part. Normally, in most electrical circuits, we can think of the inductance as being fixed. However, in the trip coil, as the plunger moves the reluctance of the magnetic circuit is reducing which means the inductance in the electric circuit is increasing. The applied voltage, which is a constant, (apart from a small voltage drop) is proportional to the rates of change of both current and inductance. So, as the rate of change of inductance goes up the rate of change of current must go down, and vice-versa. Hence an instantaneous drop in the rate of rise of the inductance, due to the plunger striking the latch or the end-stop, will cause an instantaneous rise in the rate of change of current.

(A) Coil energised, plunger (C,D) Plunger strikes latch (E) Plunger strikes buffer

(F) Main contacts open

(G) Auxiliary contacts open

(H) Trip coil de-energised

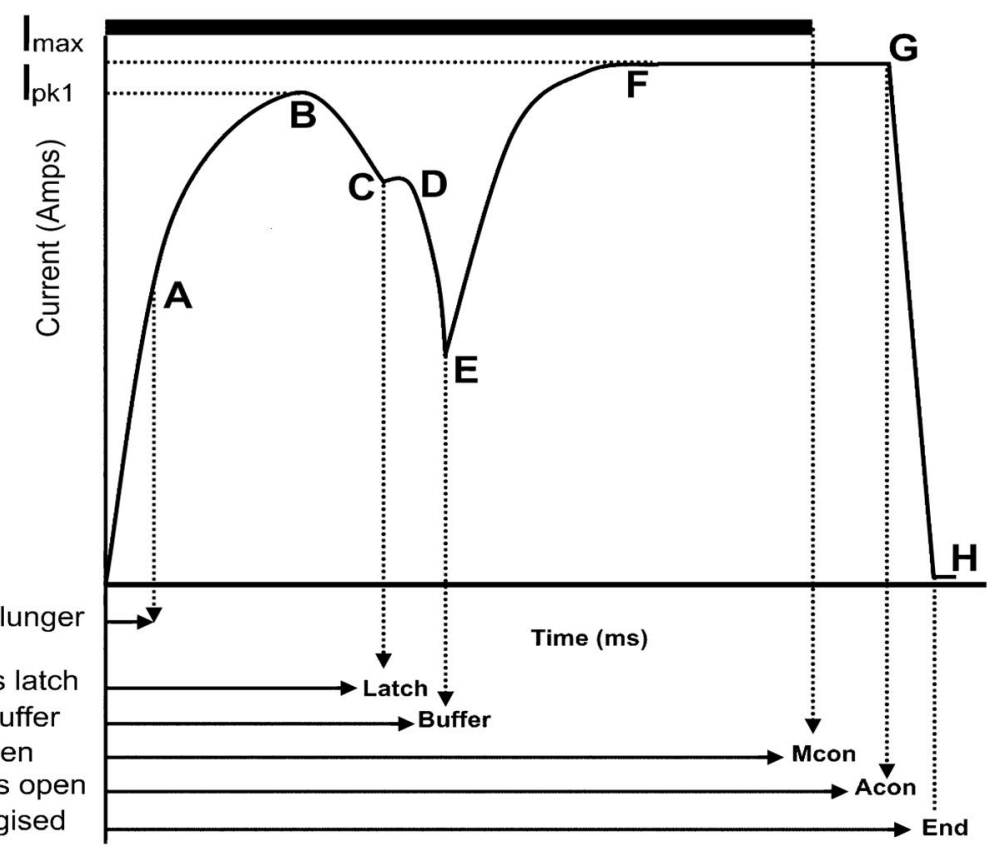

Fig. 1

Fig. 1 shows a typical trip coil profile with its distinct shape where each stage of the trip coil mechanism can be identified through its operation and problems identified if the profile deviates from a 'signature' trace.

Table 1 provides examples of what would cause a deviation in any section of the profile curve. These examples are not exhaustive and can be simulated on a trip coil mechanism by varying the parameters that cause deviations, capturing the current profile and overlaying it on a 'signature' profile. Each type of circuit breaker will have its own characteristic profile and therefore it is essential that this 'signature' profile is acquired. Comparisons can then be made with profiles captured during onsite testing of the first trip operation and subsequent trip operation to identify potential defects. 
Table 1

\begin{tabular}{|l|l|c|}
\hline DESCRIPTION & $\begin{array}{l}\text { REASON FOR DEVIATION } \\
\text { FROM STANDARD }\end{array}$ & EXAMPLES \\
\hline Zero to A & $\begin{array}{l}\text { Problem with electrical characteristics } \\
\text { of trip coil or supply voltage }\end{array}$ & $\begin{array}{l}\text { Circuit Breaker fitted with } \\
\text { incorrect trip coil (should } \\
\text { operate at } 85 \% \text { nominal) }\end{array}$ \\
\hline $\begin{array}{l}\text { A-B-C } \\
\text { Plunger and striker pin travel and } \\
\text { contact is made with trip bar }\end{array}$ & $\begin{array}{l}\text { Restrictive forces impede travel of } \\
\text { plunger/striker pin }\end{array}$ & $\begin{array}{l}\text { Oil \& greases providing a } \\
\text { dashpoint action }\end{array}$ \\
$\begin{array}{l}\text { C-D } \\
\begin{array}{l}\text { Striker pin making contact with trip } \\
\text { bar \& overcoming inertia of latch }\end{array}\end{array}$ & $\begin{array}{l}\text { Increased resistance of trip bar/latch } \\
\text { mechanism }\end{array}$ & $\begin{array}{l}\text { Incorrect adjustment of } \\
\text { latching mechanism }\end{array}$ \\
\hline $\begin{array}{l}\text { D-E } \\
\text { Plunger, striker pin, trip bar and latch } \\
\text { move together. E represents point at } \\
\text { which plunger strikes buffer }\end{array}$ & $\begin{array}{l}\text { The trip bar is restricted throughout its } \\
\text { travel }\end{array}$ & $\begin{array}{l}\text { Misalignment of trip bar and } \\
\text { striker pin guide }\end{array}$ \\
\hline
\end{tabular}

Furthermore this information is immediately available for onsite analysis and corrective maintenance can be targeted at a defect.

Also it is becoming increasingly important to make connections within the circuit breaker cubicle to ensure that the right circuits are being monitored. Some modern circuit breakers use capacitive charged power supplies, so it is important that the current probe which monitors the DC current to the trip coil, is connected close to the trip coil.

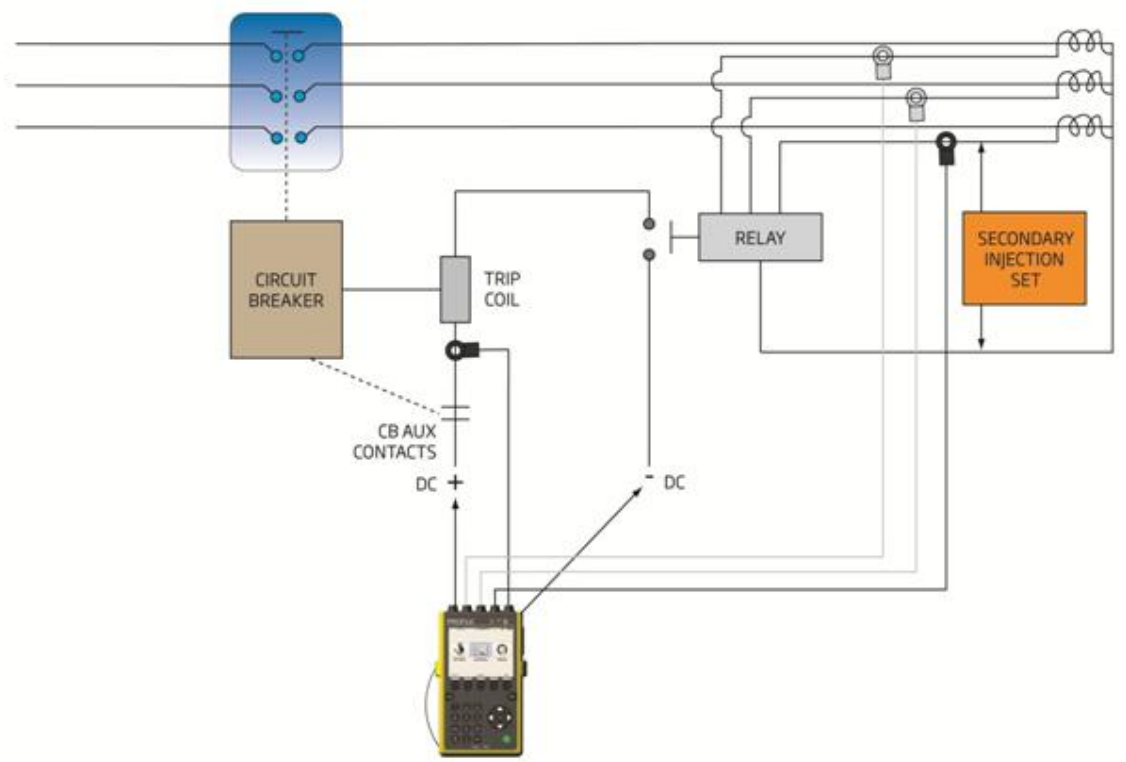

Fig. 2

Traditionally circuit breakers and protection relays are tested separately. However when a circuit breaker is called upon to operate under fault conditions, it is the combination of the protection relay and circuit breaker tripping mechanism that must operate together to ensure that the fault is isolated. Effective protection discrimination relies on the total tripping time (relay tripping time + circuit breaker operating time) operating within a set timeframe.

The measurement of the relay trip time in addition to the circuit breaker parameters as previously outlined, can be obtained by injecting current from a secondary test set to the secondary protection CT's as shown in fig. 2. 
The following are examples of problems that have been identified by capturing profiles of the circuit breaker first and subsequent trips and analysing them either directly on the Profile P3 handset or by downloading the records to proprietary analysis software called Replay Pro.

This is a good example of a circuit breaker that has a slow latch time. Notice the difference in the latch times where the past trip is about $7 \mathrm{~ms}$ faster than the present. The difference in the curves represents a trip coil that is taking longer to move the trip latch due to a buildup of friction in the release mechanism. This affects the overall circuit breaker operation, which results in a slow trip. Another supporting observation is that both curves show the same time from latch point to ACon as $11 \mathrm{~ms}$. This indicates that the problem is indeed happening before full mechanical motion of the breaker.

In this case, the slow main mechanism operation is only apparent in the first trip. The first trip MCON (Main Contact) time of $52 \mathrm{~ms}$ is well out of the acceptable range of 30 milliseconds. This was a lubrication issue on the main bearings. As you can see, the breaker is coming off latch at the same time on both trips. The second trip was taken immediately following the first, and no maintenance was performed. In this case, the breaker's grease reconstituted for a much faster operation. Most likely the mechanism will have to be disassembled for cleaning and re-greasing. Also, the first trip shows the current staying high in the trip coil longer than is required which may eventually damage the coil.

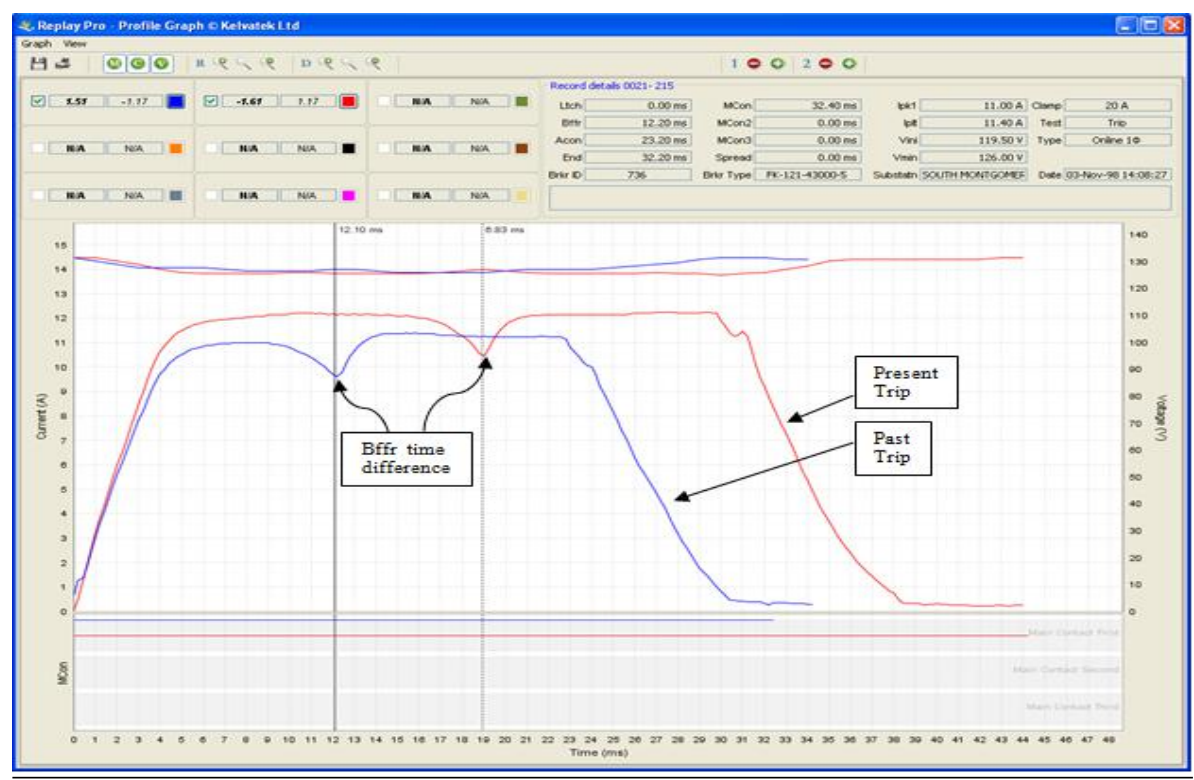

Fig. 3

In this example, the first trip operation which is indicated by the blue graph is very slow. It exceeds the maximum record length of 850 msecs and does not show any Mcon (Main Contact) values as the primary current is still present during the recording period.

The actual first trip time may well have exceeded 1 second, which would not only cause a loss of discrimination but also possible damage to the connected circuit both downstream and upstream. 


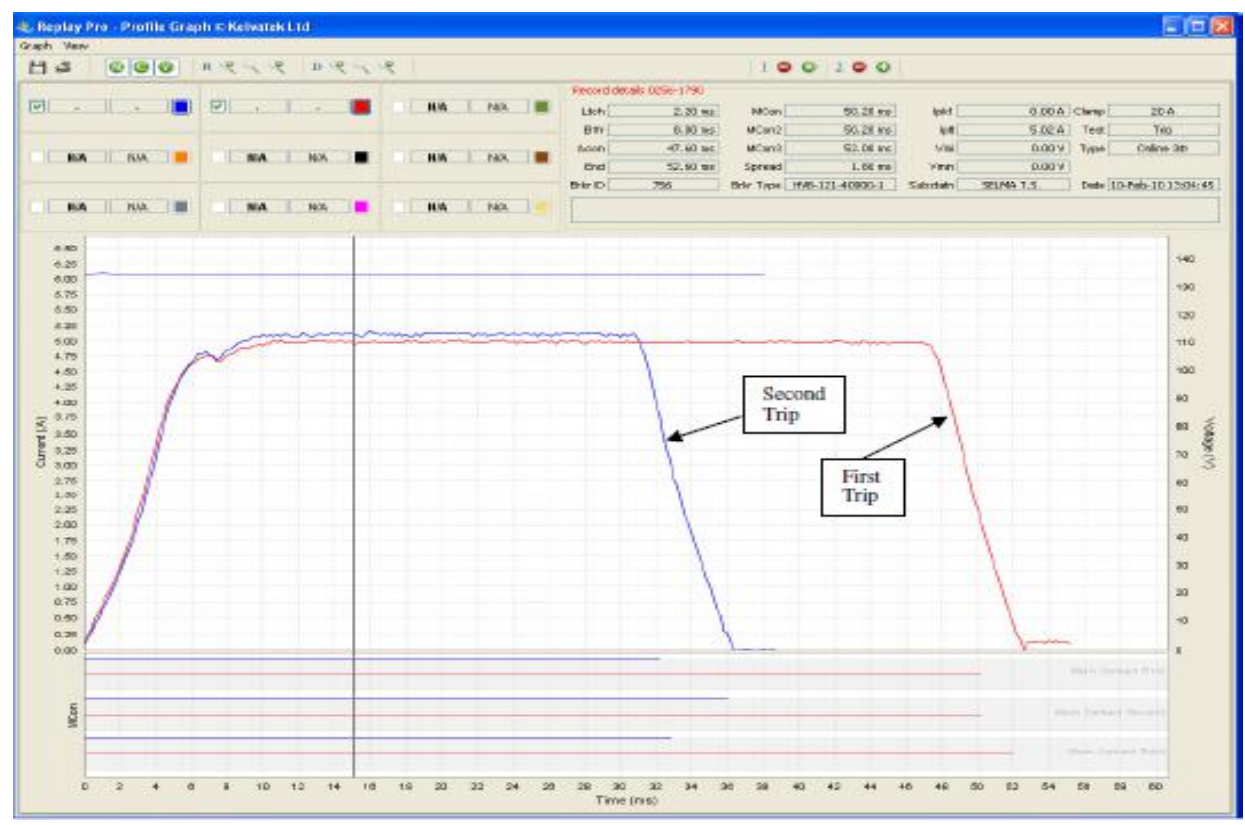

Fig. 4

There are a range of problems that are indicated here which show up on two subsequent trip operations. These include; possible battery charger fault indicated by the ripple on the DC waveform, poor contact in trip coil DC circuit and possible problems with the trip coil 'slug' sticking.

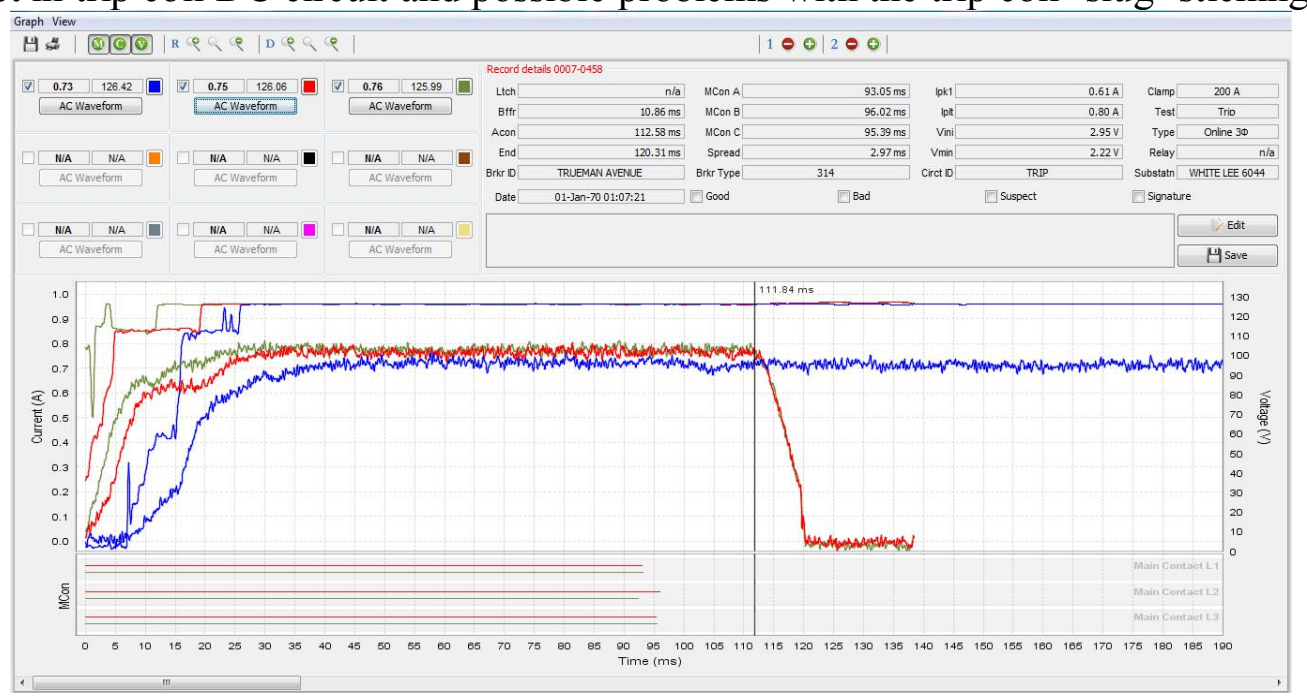

Fig. 5

The first trip which is indicated by the blue graph shows a momentary drop in the DC supply to the trip coil which results in a $6 \mathrm{msec}$ delay in the first stage of the trip coil operation. This delay is also reflected in the overall main contact time operation. However in the second trip this problem has 'cleared' with a consequential reduction in main contact time. This clearly demonstrates the importance of capturing the first trip operation where potential problems occur which are temporarily cleared during the second trip operation. 


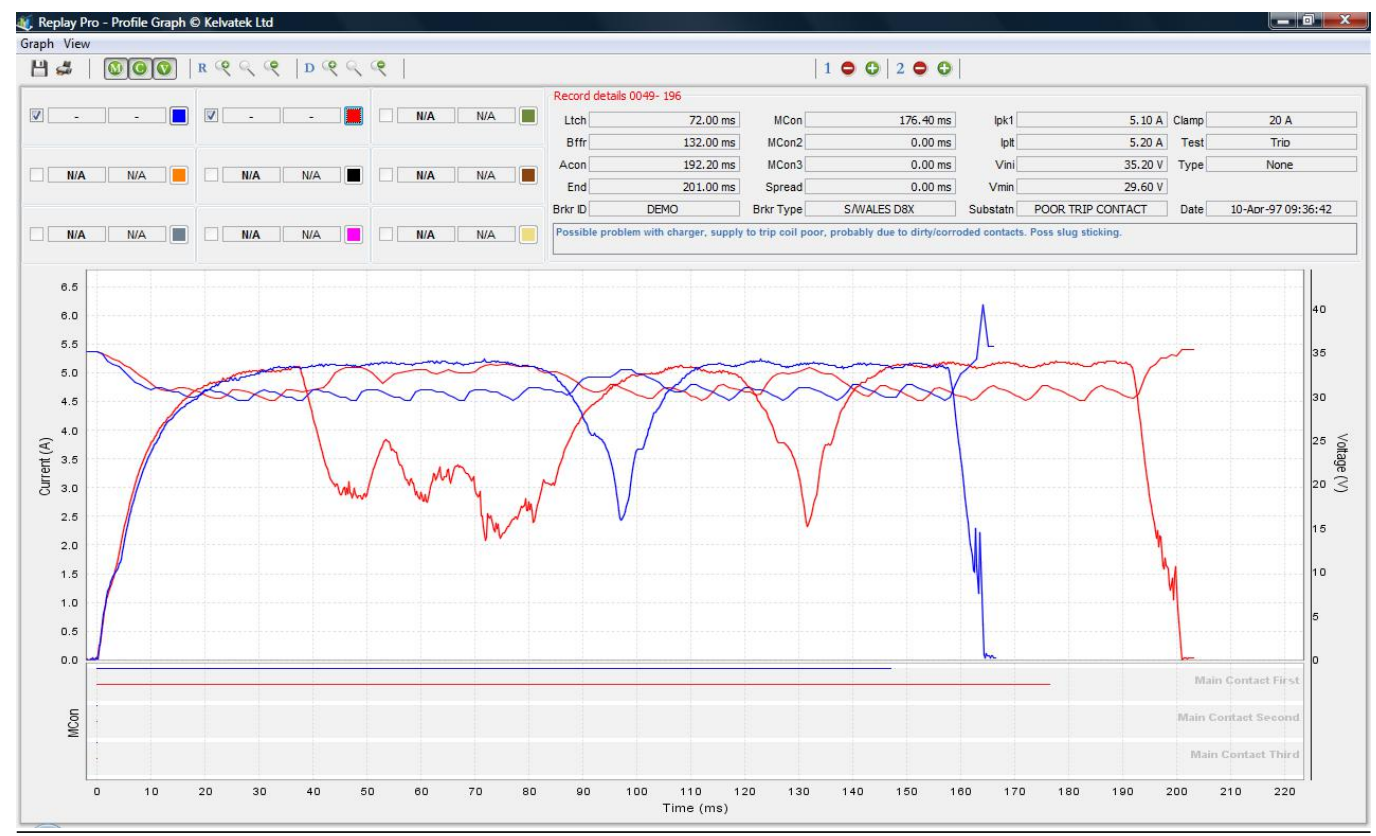

Fig. 6

\section{Conclusion}

Trough the experiments and operation of the switch's open and close, and the feature spectrum of the secondary loop current curve changing, we learned the whole process of changing of the switch's mechanical feature.

That is, to determine the mechanical malfunction of the switch from the change of secondary loop current curve is feasible and effective. The secondary loop current curve is like "ECG" and the mechanical feature is like "muscle", where there is clear correspondence.

However, the key technology is that we need to establish a database of the typical defects of the switch's secondary loop current curve pattern, in order to accurately determine the switch's mechanical malfunction.

At present, the main domestic circuit-breaker manufactures are Western Cape, Ping Gao, Shen Kai, $\mathrm{Ru}$ Gao and Tai Kai, as well as ABB, Siemens, Alstom and other joint venture manufactures. There are many various circuit-breaker manufactures and they commonly use springs, hydraulic, pneumatic, hydraulic spring, etc. Faced with so many circuit-breaker manufactures and complex equipment type, to establish a curve pattern database of typical mechanical defect, we need a lot of laboratory and field work.

\section{References}

[1] Anon. Advances in circuit breaker testing and condition monitoring.IEEE transaction Vol. 7-12(2005).

[2] Barkan Petal. Methodology for monitoring condition of HVCB.CIGRE 1998, Paper 13-04.

[3] Kang Kai, LuoJitao. Research and Development of New Instrument of Measuring Mechanical Properties of High-voltage Circuit-breaker. Microcomputer and Application

[4] Chen Jianhong, Gong Zhangqi, Chen Xinxi. Research and Development of Signal Acquisition System of Opening/Closing Process of High-voltage Circuit-breaker [J]. High Voltage Technique.

[5] Huang Yulong, QianJiali. Mechanical Condition Monitor of High-voltage Circuit-breaker. Tsinghua University. 